\title{
Co-construcción de saberes, innovación social y desarrollo territorial: una experiencia quebequense ${ }^{1}$
}

\author{
Santiago Eizaguirre Anglada ${ }^{2}$; Juan-Luís Klein $^{3}{ }^{-}$ \\ Recibido: 30 de enero de 2019 / Aceptado: 27 de noviembre de 2019 / Publicado: 1 de junio de 2020
}

Resumen. El movimiento de la economía social en Quebec cuenta con una trayectoria de concertación que lo dispone al desarrollo de dinámicas de co-construcción y transferencia de saberes. Frente a la aplicación de políticas de austeridad y al desmantelamiento de las corporaciones de desarrollo económico distintas prácticas locales plantean el diálogo entre investigación y acción sobre el terreno como una clave para la movilización ciudadana y la articulación de procesos de innovación social. En el cruce entre los saberes de dichas prácticas y un nuevo paradigma epistemológico se encuentra la organización Territoires innovants en Économie Sociale et Solidaire (TIESS). El presente artículo analiza dicha iniciativa explorando sus referentes inmediatos y tomando en cuenta los condicionantes que inciden en la transferencia de saberes sobre innovación social. Como conclusión se observa que la organización del vínculo entre actores socialmente innovadores es en la actualidad uno de los principales retos del desarrollo territorial.

Palabras clave: Desarrollo territorial; Innovación social; Transferencia de saberes; Co-construcción.

Claves Econlit: F63; O10; O35.

\section{[en] Knowledge co-construction, social innovation and territorial development: a Québec experience}

Abstract. The social economy's movement in Quebec has a trajectory of agreement that provides room for the development of dynamics of co-construction and knowledge transfer. In front of the application of austerity policies and the dismantling of economic development corporations, different local practices raise the dialogue between research and action on the ground as a key point for citizen mobilization and in order to promote the articulation of social innovation processes. At the crossroads between knowledge on these practices and a new epistemological paradigm is framed the organization Territoires innovants in Économie Sociale et Solidaire (TIESS). This article analyses this initiative by exploring its immediate referents and taking into account the constraints that affect the transfer of knowledge about social innovation. In conclusion, it is observed that the organization of the link between socially innovative actors is currently one of the main challenges of territorial development.

Keywords: Territorial development; Social innovation; Knowledge transfer; Co-construction.

Sumario. 1. Introducción. 2. La co-construcción y la transferencia del conocimiento: un ingrediente de la innovación social. 3. Antecedentes en la transferencia de saberes sobre innovación social en el Québec. 4. La co-construcción de conocimiento entorno a grupos temáticos. 5. Problemáticas comunes en la "co-construcción" del vínculo y la transferencia de saberes sobre innovación social. 6. El TIESS frente a los desafíos contemporáneos del desarrollo territorial. 7. Conclusiones. 8. Referencias bibliográficas.

Cómo citar: Eizaguirre Anglada, S.; Klein, J.-L. (2020) Co-construcción de saberes, innovación social y desarrollo territorial: una experiencia quebequense. REVESCO. Revista de Estudios Cooperativos, vol. 134, e69172. https://dx.doi.org/10.5209/REVE.69172.

1 Este artículo ha sido escrito en el marco del proyecto «El rol de les iniciatives socialment innovadores en el desenvolupament de la governança democràtica, l'economia solidària i els entorns urbans educadors», dentro del programa de formación posdoctoral financiado por la Universitat de Barcelona y la Fundació Bancària de l'Obra Social de La Caixa (2016-2019). LCF/PR/PR06/11010001. El trabajo de campo fue realizado por Santiago Eizaguirre Anglada entre febrero y julio de 2018 mediante una estancia de investigación en el Centre de Recherche sur les Innovations Sociales (CRISES), en la Université du Québec à Montréal, financiada por el programa José Castillejo de movilidad al extranjero para jóvenes doctores (2017) del Ministerio de Educación, Cultura y Deporte. Los autores agradecen también al Fonds de recherche du Ouébecsociété et culture, el cual financia las actividades del centro CRISES, y a Benoit Levesque, Nancy Neamtan, Genevieve Huot, Melanie Pelletier, Vincent van Schendel y Beatrice Alain, por sus comentarios respecto a la orientación y los objetivos de la investigación.

2 Universitat de Barcelona, España

Dirección de correo electrónico: santieizaguirre@ub.edu.

3 Université du Québec à Montréal, Canadá

Dirección de correo electrónico: klein.juan-luis@uqam.ca. 


\section{Introducción}

La transferencia de saberes sobre innovación social requiere de una aproximación participada a la construcción de aprendizajes que rompe con los esquemas propios de la transferencia de conocimientos en el marco de la sociedad industrial. El énfasis en la participación y el vínculo entre actores como aquello que posibilita la innovación social desplaza la atención de la transmisión de información sobre productos o soluciones que ofrecen respuesta a problemas sociales concretos, hacia la apropiación de procesos colectivos generadores de aprendizajes integrales. Entender la innovación social como prácticas superadoras de la exclusión social que se generan desde la acción colectiva, implica el aprendizaje en un sentido social y estimula una aproximación a la investigación transdisciplinaria (Moulaert et al. 2013). La transferencia de saberes sobre innovación social requiere una nueva cultura de acompañamiento mutuo entre actores comprometidos con la transformación social (Klein, Laville y Moulaert, 2014). Esta perspectiva, que en el medio de las ciencias sociales se ha identificado con la perspectiva de la investigación-acción participativa o partenarial (Fontan, 2010, Fontan et al., 2013), ha tenido una influencia clara en el ámbito de la acción pública, y en especial en la práctica de los actores que se dedican a la economía social y al desarrollo territorial (Pecqueur y Itcaina, 2012). La emergencia de la economía social en las políticas públicas, que en un contexto de políticas de austeridad ha sido más visible en el terreno de los discursos que en el de las prácticas (Chaves-Avila y Demoustier, 2013; Chaves-Avila y Savall-Morera, 2019) va acompañada también de una cada vez mayor atención a la epistemología partenarial y su modelo organizativo.

Los actores implicados en el fomento de la economía social hacen referencia a la co-construcción de conocimientos (Vaillancourt, 2019) como el proceso de reconocimiento, reflexividad y colaboración entre sujetos implicados que facilita la transferencia de saberes y a la vez posibilita la transformación y el desarrollo territorial. Estos actores reconocen que la co-construcción de soluciones participadas a los retos sociales es tan importante, y en muchas ocasiones mucho más dificultosa que la propia identificación, paquetización y transferencia de las soluciones concretas que identificamos con la innovación social. La definición de la innovación social promovida en el entorno de las ciencias sociales, que identifica a prácticas que ofrecen respuestas a necesidades sociales que ni el mercado ni el Estado ha satisfecho; experiencias que empoderan a colectivos sujetos a dinámicas de exclusión; y/o experiencias que producen mecanismos y sistemas de tratamiento y transformación de las relaciones de poder (Moulaert et al., 2013); se completa alrededor de la idea de proceso de aprendizaje compartido cuando se observa desde la perspectiva de la transferencia de conocimientos.

La pregunta que ha guiado nuestra investigación es en qué consiste promover la transferencia de saberes sobre innovación social y como se puede garantizar la utilidad social de los procesos de co-construcción que identificamos como claves en las prácticas innovadoras a nivel local. De qué manera las innovaciones sociales en tanto que procesos de co-construcción de saberes territorializados y ligados a un contexto institucional concreto pueden dar lugar a dinámicas de transferencia de conocimiento, y en qué sentido esto tiene un valor para el entorno que se dedica al desarrollo territorial. Considerando el interés por posibilitar la escalabilidad de la innovación social en un sentido gubernativo, geográfico o social, nos preguntamos cómo se puede garantizar el valor social de la co-construcción de saberes en el ámbito de la innovación social y el desarrollo territorial. En este texto planteamos el abordaje de dicha cuestión mediante el análisis de una entidad que tiene por objeto y razón de ser la transferencia de saberes sobre innovación social y desarrollo territorial: Territoires Innovants en Économie Sociale et Solidaire (TIESS), en Quebec, Canada. Analizar el caso del TIESS nos ofrece la posibilidad de profundizar en el papel de las iniciativas de innovación social en el fomento la gobernanza democrática y la economía social y solidaria (ESS). Esta entidad subraya las iniciativas de innovación social como entornos de co-creación, vínculo entre actores y espacios de transferencia de saberes. Recoge la herencia de la investigación-acción partenarial, asume como responsabilidad el ofrecimiento de respuestas a los problemas que surgen con la puesta en marcha de las dinámicas de co-construcción, y en hacerlo da lugar a la reflexión significativa sobre los retos contemporáneos del desarrollo territorial y la economía social.

La globalización neoliberal, con su minimización de los sistemas de gobernanza públicos (New Public Management), que, como describe Naomi Klein (2016), fue experimentada en Chile y luego difundida en el resto del mundo, se ha visto acentuada por la aplicación de ajustes de austeridad. Las políticas de contención del gasto público aplicadas a partir de los 80s e intensificadas con el avance de la Gran Recesión a partir de 2008, han supuesto la supresión de muchas instancias de apoyo a los procesos territoriales de desarrollo económico. El contexto territorial que abordamos en este texto es el de Quebec, una provincia canadiense que ha puesto en práctica desde los años 60 un modelo de gobernanza social-demócrata que la distingue del resto de Canadá y de América del Norte. En este modelo, las asociaciones y las empresas de economía social juegan un papel importante tanto como complemento de la acción pública como en cuanto a expresión de las aspiraciones de autonomía de la sociedad civil (Bouchard, 2013). Se puede decir que el modelo de gobernanza quebequense es un modelo híbrido que combina las esferas privada, pública y social (Klein et al. 2017). 
A pesar de la influencia de la economía social en el Quebec, el Partido Liberal, que gobierna esta provincia desde el 2003 de manera casi ininterrumpida, procedió en el año 2015 al desmantelamiento de las instancias de acompañamiento e intermediación que trabajaban en el campo del desarrollo territorial con una perspectiva integral ligada a la creación de dinámicas de innovación social. Exacerbando un enfoque meramente economicista de los procesos de desarrollo territorial, el gobierno del Partido Liberal impulsó el desmantelamiento de estructuras como las Corporations de Développement Économique Communautaire (CDEC), los Centres locaux de développement (CLD) o los Conseils régionaux des élus (CRE), además de varias otra agencias regionales y locales que habían sido iniciadas desde la base. Estas estructuras realizaban hasta entonces un trabajo de vertebración territorial y comunitaria clave en la concertación de un desarrollo económico integrado y socialmente creativo ${ }^{4}$.

La desaparición de estos organismos dejó un vacío que ha interpelado especialmente al entorno de la economía social, así como a los investigadores comprometidos con el desarrollo territorial. Los actores implicados, incluyendo a las administraciones locales y las organizaciones comunitarias, se han reagrupado alrededor de nuevos dispositivos más o menos formalizados. Por un lado, los polos territoriales de fomento de la economía social ${ }^{5}$ promovidos por el Chantier de l'Economie Sociale ${ }^{6}$ han mantenido vivo el trabajo en red entre actores sobre el terreno, pero también las organizaciones orientadas a promover la transferencia de conocimientos entorno a la innovación social se han convertido en canales de transmisión de conocimiento (Klein, Laville y Moulaert, 2014). En esta dinámica de reestructuración frente a la ofensiva neoliberal, queremos analizar la emergencia y el posicionamiento de la iniciativa Territoires Innovants en Économie Sociale et Solidaire (TIESS), una iniciativa que se inscribe en un ecosistema caracterizado por la concertación entre movimientos sociales, sindicatos, organizaciones comunitarias, universidades y administraciones públicas (Klein et al., 2013; Levesque, 2016).

La investigación se ha asentado en la metodología del estudio de caso y ha utilizado como principal herramienta de recogida de datos a la entrevista en profundidad. El trabajo de campo se realizó entre febrero y junio de 2018 en Montreal (Quebec) mediante la realización de una estancia de investigación en el Centre de Recherhche sur les Innovations Sociales (CRISES) en la Université du Québec a Montréal. Durante la estancia se realizaron un total de doce entrevistas en profundidad a actores representantes de las distintas entidades de fomento de la innovación social en el Quebec (Chantier de l'Économie Sociale, Fiducie du Chantier, CITIES Collaborations en économie sociale et solidaire, TIESS) y a referentes del entorno académico que estudia la economía y la innovación social en el territorio (CRISES, CIRIEC-Canadà). También se participó en distintas visitas a experiencias de desarrollo territorial (CDEC LaSalle-Lachine, Municipalité Canton de Saint-Camille, Ilot Pelletier a Montréal Nord de Parole d'ExcluEs). En consonancia con el enfoque de la investigación partenarial se participó activamente en seminarios y talleres promovidos por las iniciativas que aparecen citadas a lo largo del texto (Incubateur universitaire Parole d'ExcluEs) y en un coloquio científico dedicado a los retos de futuro del desarrollo territorial. Entre las actividades complementarias a las entrevistas en profundidad que tuvieron especial relevancia para la sistematización de los resultados cabe señalar la participación en una sesión del consejo científico del TIESS, así como en la jornada de planificación estratégica anual de la entidad en abril de 2018.

El artículo arranca con una breve exposición de la problemática de la transferencia de saberes y de la coconstrucción del conocimiento en el marco del desarrollo territorial. Luego, se exponen los antecedentes de la transferencia de conocimientos sobre innovación social en el Quebec. Se analizan tanto entidades del ámbito académico como iniciativas experimentales que han dado importancia a la transferencia de saberes sobre innovación social y dan sentido a la labor desarrollada por el TIESS. A continuación, el artículo presenta la metodología específica de trabajo del TIESS abordando las actividades y productos generados por los grupos de trabajo en los que se organiza. A este respecto se observa como la disparidad temática en la que se mueve la entidad demanda de la reflexión estratégica para formalizar y dar coherencia a su metodología. A continuación se presentan las problemáticas comunes en el fomento del vínculo y la transferencia entre actores provenientes de distintos ámbitos profesionales y geográficos. En un último apartado se sitúa el estudio de caso en relación a los retos contemporáneos del desarrollo territorial. Las conclusiones sostienen que la transferencia de saberes sobre innovación social requiere apuntar hacia modelos des-mercantilizados de organización del vínculo creativo entre actores socialmente comprometidos.

Con respecto a la creación y a la función de estos organismos, se puede consultar el artículo Klein et al. (2003)

Los Pôles d'Économie Sociale son 22 polos presentes en todas las regiones del Quebec que tienen la misión de promover el sostén y el desarrollo de la economía social regional.

6 En lo que respecta al Chantier de l'économie sociale, ver: https://chantier.qc.ca/ 


\section{La co-construcción y la transferencia del conocimiento: un ingrediente de la innovación social}

Aparte de situarse en un momento de sacudida a nivel político e institucional debido al desmantelamiento de las instancias de gobernanza del desarrollo territorial, la implantación del TIESS debe ser enmarcada también en un contexto global de giro epistemológico por lo que respecta a la importancia del conocimiento y de la reflexividad en el dominio del desarrollo territorial (Klein, 2017a). Este cambio de paradigma implica modelos de investigación en partenariado donde académicos y actores locales reflexionan de manera conjunta acerca del tipo de desarrollo que contribuye a la prosperidad colectiva (Gilet y Tremblay, 2017). Un momento en el que el reconocimiento de la investigación colaborativa y el replanteamiento de la utilidad social de la actividad académica se identifican en tanto que imperativos epistémicos (GUNI, 2017).

La implantación de una organización de transferencia del conocimiento que tiene por objeto específico la co-construcción de conocimientos apropiados para generar innovación social puede relacionarse con un cambio en el pensamiento respecto a la utilidad y aplicación del conocimiento generado por las ciencias sociales en un contexto de crisis. Al margen que la co-construcción y la transferencia de saberes son cuestiones en las que la literatura sobre investigación-acción partenarial ya hace tiempo que indaga (Fontan et al, 2014; Dumais, 2011; Longtin, 2010; Gillet et Tremblay, 2017), resulta evidente que su presencia en la agenda de investigación pública está relacionada con un cambio sistémico global.

Como ha sido establecido en un texto anterior (Klein, 2017a), la innovación social se basa principalmente en un proceso de aprendizaje colectivo. La referencia constante a la innovación social en el mundo actual no se trata simplemente de una moda pasajera, sino que va ligada a la existencia de una estrategia de desarrollo alternativo al capitalismo hegemónico basada en valores socialmente transformadores. Sin embargo, las innovaciones sociales en si no garantizan la implantación de un modelo alternativo de desarrollo a menos que están arraigadas en una perspectiva ciudadana que permita avanzar hacia una transformación social más integral y completa. El trabajo colaborativo entre investigadores y actores puede contribuir a este avance mediante la co-construcción de nuevos conocimientos a través de la experimentación social. El reto es generar y difundir conocimientos que sean relevantes tanto para la comprensión adecuada de los problemas que afectan a las comunidades como para guiar a los actores en la puesta en práctica de estrategias adecuadas.

Se trata de un cambio de paradigma que implica una nueva manera de entender los procesos de innovación social y desarrollo territorial (Torre, 2015). El planeamiento estratégico cada día más busca romper con un abordaje de arriba abajo (top-down) en los procesos de desarrollo económico, concebidos principalmente en torno a acciones de formación, acompañamiento e intermediación replicables de un territorio a otro. Son cada vez más apreciadas como herramientas de desarrollo territorial la co-construcción y la transferencia de conocimientos sobre innovación social de abajo arriba (bottom-up) o ligada a la base (bottom-linked) (Eizaguirre et al., 2012; Novy et al., 2012; Parés et al., 2017, Klein, 2017a). La literatura especializada ha resaltado la importancia de una aproximación comprometida con la co-construcción del conocimiento sobre innovación social. La hipótesis subyacente es que la atención a la construcción de vínculos y la transferencia de saberes entre actores socialmente creativos tiene efectos prácticos a nivel local en la mejora de las condiciones de vida de las comunidades más vulnerables y en general en la democratización socioeconómica de las sociedades contemporáneas (Oosterlynck et al., 2013). En esta dirección en la siguiente sección apuntamos entre otras experiencias dos ejemplos de iniciativas locales que consideramos significativas porque inspiran la acción del TIESS: una comunidad rural implicada en el desarrollo territorial que ha dado valor a la transferencia de saberes y a la construcción de un relato común entre actores socialmente creativos (Atelier de Savoirs Partagés en Saint-Camille); y una organización comunitaria en un barrio desfavorecido de Montreal que ha dado importancia también a la transferencia de saberes y el vínculo con el ámbito académico como herramienta de superación de las dinámicas de exclusión (Incubateur universitaire Parole d'ExcluEs en Montréal-Nord).

\section{Antecedentes en la transferencia de saberes sobre innovación social en el Quebec}

En el Quebec, desde hace más de 20 años, los actores del desarrollo territorial sobre el terreno y los universitarios que se dedican a la investigación sobre economía social y desarrollo intercambian puntos de vista y trabajan de manera conjunta con el fin de producir y "difundir nuevos conocimientos. Esta colaboración se consolidó de manera notable en el marco de dos iniciativas que son referentes para el sector: por un lado, el Chantier de l'économie social, nacido el 1996; y por el otro el Centre de recherche sur les innovations sociales (CRISES), creado en 1986. Estas instituciones, con la contribución del Service aux collectivités de l'UQAM y el Institut Karl Polanyi de l'Université Concordia, dieron lugar a la creación del TIESS. 
El Chantier de la economía social es la entidad que se dedica a la promoción y la representación de la economía social y solidaria en el Quebec ${ }^{7}$. Su misión es promover la ESS como una parte integral de la economía del Quebec y a la vez participar en la democratización de la economía, así como en la emergencia de un modelo de desarrollo basado en los valores de la solidaridad, la equidad y la transparencia. El desarrollo de una iniciativa como la del TIESS debe enmarcarse también en la experiencia previa de dichas entidades en redes como l'Alliance de recherche universités-communautés en économie sociale (ARUC-ES) (2000-2010) y la Réseau québécois de recherche partenariale en économie sociale (RQRP-ÉS) (2005-2011) (Fontan, 2010). A partir de 2001, las organizaciones que forman parte de estas redes de investigación buscaron una manera de consolidar y estabilizar los frutos de su colaboración. En dos ocasiones dichas iniciativas plantearon proyectos a las instancias gubernamentales con el objetivo de desarrollar un centro de transferencia sobre innovación social específicamente promovido por el sector de la economía social y solidaria. En la "Estrategia quebequense para la investigación y la innovación" (Stratégie québécoise de la recherche et l'innovation) adoptada por el gobierno provincial en el 2010, se estableció el apoyo a la creación de organismos de vínculo y de transferencia en innovación social (Organismes de liaison et de transfert en innovation sociale-OLTIS). En 2011 siguiendo una convocatoria del Ministerio de Développement économique, de l'innovation et de l'Exportation del Quebec para financiar un nuevo organismo de vínculo y transferencia en innovación social ${ }^{8}$, las organizaciones citadas anteriormente presentaron la propuesta que finalmente culminó en la creación del TIESS. En junio de 2012 el proyecto obtuvo el reconocimiento por parte del ministerio y la financiación fue confirmada durante el otoño de 2013. El TIESS tuvo su asamblea de fundación el 20 de noviembre de 2013 en la que se juntaron más de sesenta organismos de los medios de la educación superior, de la economía social y del desarrollo territorial.

Para explicar su génesis como iniciativa de vínculo y transferencia hace falta resaltar que el TIESS es una iniciativa que se ha articulado de manera progresiva en base a una historia de concertación entre una gran diversidad de actores: universidades, agentes de economía social, municipios, sindicatos y agentes promotores de finanzas socialmente responsables. En dicha articulación, el papel de la investigación-acción participativa desarrollada desde el entorno universitario tiene una centralidad importante. Es en este sentido que es interesante observar los antecedentes de colaboración entre el entorno universitario y los actores sobre el terreno en el ámbito de la transformación social, sobretodo presentados en el entorno del Quebec alrededor del centro de investigación CRISES (Lévesque et al. 2014).

El CRISES a lo largo de los años noventa se constituyó como un centro multi-universitario y multidisciplinario que actúa con el Quebec como laboratorio de análisis e intervención. Progresivamente el grupo se ha ido transformando más allá de un espacio de análisis en una aproximación científica que ha dado lugar a una escuela de pensamiento que trata las transformaciones societales inducidas por los procesos de innovación social (Klein \& Fontan, 2014: 3). La aproximación del Centre de Recherche sur les innovations sociales (CRISES) da un lugar central a la investigación partenarial y sitúa la cuestión de la transformación social en el corazón de la reflexión científica (Klein, 2017b). Como destacan Bouchard \& Levesque (2010) los trabajos realizados en el marco del grupo se enmarcan en tres corrientes teóricas: la aproximación a los movimientos sociales, la teoría de la institucionalización (con la teoría de la regulación y las convenciones) y

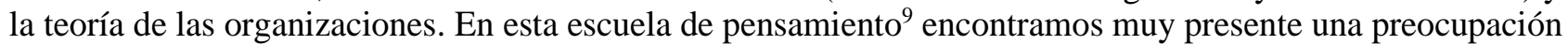
por construir una aproximación útil y compartida con la visión de distintos actores colectivos (sindicatos, movimiento comunitario, movimiento cooperativo y economía social, entre otros) y una interacción con dispositivos abiertos a dichos actores como pueden ser el Service aux collectivités de l'UQAM o el Centre interdisciplinaire de recherche et d'information sur les entreprises collectives (CIRIEC- Canadà). La preocupación por ofrecer una investigación transformadora se manifiesta en el caso del CRISES en la producción de herramientas teóricas y metodológicas que responden a las exigencias del mundo académico y a la vez una producción susceptible de contribuir al cambio social ofreciendo análisis desde perspectivas pertinentes para los actores colectivos vinculados al terreno (Klein, 2017b).

Como ejemplos prácticos de la perspectiva descrita, en el Quebec hay distintas experiencias de investigación-acción partenarial que han dado importancia a la transferencia de conocimientos mediante una actividad científica que interviene en el entorno. Estas experiencias tienen un papel importante en tanto que iniciativas que inspiran y se nutren de la transferencia promovida por el TIESS. Son iniciativas que han sido

Según la web del Chantier en 2018 la economía social en el Quebec constaba alrededor de más de 7000 empresas colectivas acumulando globalmente una cifra de negocios que superaba los 40000 millones de dólares, por delante de los sectores de la construcción, la aeronáutica y la explotación minera juntos. Más de 210000 personas trabajaban en el entorno de la economía social en el Quebec, en todos sus sectores de actividades, del comercio al detalle a las nuevas tecnologías (https://chantier.qc.ca/).

8 En un principio se crearon dos en campos específicos, la educación (https://www.ctreq.qc.ca/) y la intervención y la prevención psicosocial (http://www.clipp.ca/). Al término del año 2011, el gobierno lanza un llamado a postulaciones para crear un tercer OLTIS, al cual postularon los fundadores del TIESS.

9 Partiendo de dicha pluralidad de perspectivas teóricas, y desde las distintas perspectivas disciplinarias dentro las ciencias sociales que se aproximan a ellas, las actividades de investigación de los miembros del CRISES están organizadas en torno a cuatro ejes de análisis de las innovaciones sociales y las transformaciones: 1) en las políticas y las prácticas sociales; 2) en el territorio y las colectividades locales; 3 ) en las empresas colectivas; 4) en el trabajo y el empleo. 
documentadas en distintas publicaciones como ejemplos de co-construcción de saberes entre el entorno académico y actores sobre el terreno.

Saint-Camille, por ejemplo, es una comunidad rural, localizada en el sur de la provincia de Quebec conocida porque durante las últimas dos décadas ha protagonizado un proceso de dinamización comunitaria y desarrollo económico ejemplar. Esta localidad ha hecho frente al declive demográfico, abordando temas como la dinamización cultural y comunitaria, el ofrecimiento de vivienda asequible, la rehabilitación y reutilización del patrimonio, o el impulso económico a iniciativas colaborativas vinculadas a sectores estratégicos. Observando estas dinámicas, miembros del CRISES, en colaboración con la comunidad local implicada en el desarrollo territorial, han desplegado una experiencia denominada Ateliers de savoirs partagés (Talleres de saberes compartidos). En el contexto de estos talleres, líderes comunitarios y vecinos, durante más de dos años (2012-2014), compartieron con investigadores sociales la construcción de un relato común sobre el proceso de desarrollo económico protagonizado por el territorio. Esta experiencia ha puesto de relieve las principales componentes del modelo de acción de la localidad de Saint-Camille ${ }^{10}$, con el objetivo, por un lado, de transmitir dicho modelo a nuevos residentes de esta comunidad, y por el otro lado, de identificar los elementos susceptibles de inspirar otras comunidades que pueden tener la necesidad de desarrollar procesos de revitalización similares (Klein et al., 2015). La transferencia de conocimiento promovido alrededor de dicha iniciativa puede analizarse como un referente claro del TIESS.

Otro ejemplo de iniciativa de innovación social que pone en marcha un proceso de investigación-acción partenarial en el que miembros del CRISES han tenido un papel relevante y que también nutre la metodología aplicada por el TIESS ha sido la iniciativa Parole d'ExcluEs en la zona Norte de la ciudad de Montreal. Parole d'ExcluEs es una organización independiente creada en 2006 con la intención de renovar las prácticas de lucha contra la pobreza y la exclusión social urbana. La iniciativa empezó en el Îlot Pelletier en Montreal-Nord (2007), fue seguida por una segunda promoción de vivienda comunitaria en la antigua fábrica de galletas Viau en Mercier-Hochelaga-Maisonneuve (2008) y se adaptó también en otro sector desfavorecido de Montreal-Nord (2013). Establecida en la actualidad en estos tres sectores desfavorecidos de la ciudad de Montreal, la organización ha desarrollado un modelo de acción que parte de la atención a las necesidades expresadas por las personas que viven en situación de exclusión social y de pobreza y que da una importancia clave a la movilización ciudadana para su tratamiento y transformación. Dando coherencia estratégica a una gran diversidad de acciones y apelando a la investigación-acción partenarial, la coconstrucción del conocimiento y el cruce de saberes se creó a su alrededor la incubadora social universitaria Parole d'ExcluEs. Desde 2007 la incubadora en cuestión es una pieza clave en tanto que espacio de reflexión alrededor de problemas y planteamientos derivados del acompañamiento de proyectos de inclusión social. Con la inspiración de proyectos de desarrollo social en el Brasil en los que la investigación juega un rol importante en el acompañamiento al desarrollo comunitario (Dagnino, 2012 Dubeux, 2013; Fernandez et al. 2013; Pozzeborn, 2015), Parole d'ExcluEs ha alimentado desde sus inicios un dialogo continuo con investigadores comprometidos con la investigación-acción partenarial en el Quebec. Esto por un lado incluye la realización de distintos estudios sobre las necesidades y las aspiraciones de los vecinos del entorno en el que se sitúa la iniciativa. También la puesta en funcionamiento de un laboratorio sobre el cruce de saberes, la elaboración de una guía sobre las prácticas de Parole d'ExcluES y la contribución a proyectos experimentales concretos como el desarrollo de una clínica de proximidad o el impulso de un sistema alimentario para todos los ciudadanos de los entornos urbanos en los que se sitúa la experiencia.

Mediante la expresión "desarrollo del poder de actuar" (développement du pouvoir d'agir), en el entorno de la incubadora universitaria Parole d'ExcluEs, investigadores y actores se preguntan de qué manera la iniciativa ha permitido reforzar la cultura de movilización, ya sea entre la ciudadanía dentro del barrio como entre los distintos organismos e entidades que dan soporte a la iniciativa. También la consideración del concepto "desarrollo del poder de actuar" se orienta a observar cómo esto ha conducido a cambios estructurales ya sean a la escala del barrio o de la municipalidad (Heck, 2017; Le Bossé et al. 2017; Fontan et al. 2017).

\section{La construcción de conocimiento en torno a grupos de trabajo temáticos}

Influenciada por las experiencias anteriores, la tarea del TIESS se centra en identificar, inventariar y sistematizar las innovaciones experimentadas por las empresas colectivas y las organizaciones comunitarias que trabajan específicamente en el ámbito de la economía social y solidaria. Con el fin de favorecer la difusión y la apropiación de nuevos mecanismos y maneras de hacer, la entidad promueve la construcción de vínculos entre los actores del sector y más allá de este. Su actividad se orienta por tanto a promover la transferencia de conocimientos prácticos y teóricos co-construidos por los profesionales de la economía

10 En el sitio http://recitsrecettes.org/ateliers se pueden consultar todos los materiales y los resultados de la realización de esta experiencia. 
social, así como por los investigadores que la estudian Los objetivos principales de la entidad, tal y como se presentan en su web son: 1) estimular los actores comprometidos en construir de manera conjunta nuevos conocimientos; 2) asegurar la difusión y la transferencia de conocimientos; y 3) enriquecer las prácticas de los investigadores y de los profesionales de la economía social con el objetivo de impulsar el desarrollo de los territorios. Estos objetivos se articulan alrededor de la organización de actividades y el desarrollo de productos que promueven la transferencia de conocimientos y prácticas. En concreto, el TIESS no desarrolla específicamente investigación, sino que se centra en tejer alianzas entre los actores de la economía social y el mundo de la investigación para construir nuevos conocimientos y aplicarlos a la resolución de problemas relacionados con el desarrollo territorial. La aproximación del TIESS a la transferencia de conocimientos se basa en el reconocimiento que los saberes académicos y prácticos pueden complementarse para hacer frente a los retos sociales. El mandato de la iniciativa se concreta en promover el vínculo, la vigilia y la transferencia de innovaciones sociales en el sector de la economía social. Por lo que respecta al vínculo se parte del hecho que la innovación social dentro del sector de la economía social y solidaria es a menudo el fruto del trabajo de las organizaciones y sus miembros. Apoyándose sobre las redes existentes tanto de profesionales del sector como de investigadores interesados por este, y partiendo siempre de la voluntad de aprovechar las sinergias de sus iniciativas y competencias, las acciones del TIESS buscan desarrollar y reforzar los lazos entre todos los actores implicados.

La metodología del TIESS gira alrededor de la construcción de grupos de trabajo que reúnen a investigadores y profesionales interesados por una temática particular. Estos grupos de trabajo son comisiones que combinan el mandato de construir un estado de la cuestión sobre una temática en concreto con el reto de alimentar el vínculo y la transferencia dentro del sector. Esto incluye tareas vinculadas a identificar las necesidades del sector, una observación detallada de su evolución histórica en relación a la temática en cuestión, promover actividades y herramientas de transferencia como webinars y seminarios de divulgación, y en, algunos casos, el desarrollo de proyectos autónomos. Uno de estos proyectos autónomos es la edición de una segunda etapa de los Ateliers de savoirs partagés, ya promovidos en Saint-Camille entre los años 2012 y 2014. En la actualidad, aparte de ser re-editados en Saint-Camille, están siendo replicados en otras dos comunidades rurales distintas (Petit-Saguenay en la región de Saguenay-Lac-Saint-Jean y MRC Belchasse en la región de Chaudièrre-Appalaches). Esta nueva edición del proyecto Ateliers de savoirs partagés empezó a desarrollarse en 2018 y está prevista hasta el 2021.

Entre los primeros grupos de trabajo desarrollados por el TIESS encontramos uno que profundizó en la relación entre la enseñanza superior y el desarrollo de territorios, sistematizando los modelos de colaboración con el medio de la economía social. Esta comisión se orientó a valorar la contribución a la formación en economía social que se podía hacer en el contexto de la educación superior, incluyendo las universidades y los colegios post-secundarios (cégeps). En este caso, el trabajo en grupo sirvió para el desarrollo de nuevos contenidos y formaciones y para dar respaldo a las iniciativas de las redes presentes que se encontraban actuando en esos dominios. Otro grupo de trabajo que se ha desarrollado en el marco del TIESS es sobre nuevos mecanismos de financiación. Mediante la participación de organismos de finanzas solidarias, de la economía social y del ámbito de la investigación, este grupo analizó la financiación participativa, la utilización de capitales provenientes del sistema de pensiones y la emisión de obligaciones comunitarias (Beaudin et al., 2016).

Como productos derivados de los grupos de trabajo, el TIESS cuenta con distintas síntesis de conocimientos que se convierten en herramientas de transferencia. La primera de estas síntesis versa sobre el emprendimiento social y sus distintas formas de comprensión (Roy, 2016). En base al trabajo en seminarios con miembros del sector de la economía social se ofrece una aproximación detallada a la conceptualización compleja de un concepto borroso. El objetivo en este caso era dilucidar las diferencias, aspectos dudosos y puntos en común entre la noción de economía social y la de emprendimiento social, divulgando un lenguaje compartido entre actores.

Otra de las síntesis de conocimientos realizadas en el marco del TIESS se encuadra en un proyecto orientado a acompañar y documentar el desarrollo de un modelo de franquicia adaptado a la realidad de las empresas de economía social. Dicha propuesta se entiende con el fin de explorar los condicionantes que pueden facilitar la replicación de una innovación en varios territorios (Huot et al., 2016). En concreto se orienta a ayudar a las empresas de economía social a identificar si la franquicia es una fórmula de cambio de escala que se adapta a su situación particular concreta. En el caso de este grupo temático fue la demanda de asesoramiento al TIESS por parte de una empresa en concreto lo que dio lugar a la creación de un grupo de trabajo.

El TIESS también ha dado importancia a la movilización de actores en el contexto de la mediación cultural. En este caso se ha interesado por reconocer las ambigüedades relativas a la noción de mediación cultural aplicada al desarrollo de los territorios. Dentro del contexto general de la descentralización de las políticas culturales, se ha explorado la cuestión de como movilizar a los agentes, que estrategias utilizar, y cómo proceder en la promoción de la concertación territorial. Dicha síntesis de conocimientos se ha orientado a los agentes que tienen el reto de coordinar a una diversidad de instancias alrededor de un 
proyecto de índole cultural. Cuestiones como el escalamiento de la estrategia, la valorización de la misión y el campo de acción de los proyectos, así como la afirmación de la legitimidad de estos, se han considerado claves como estrategias en la movilización de actores (Lusier, 2016: 4).

Hasta aquí hemos observado en líneas generales las principales temáticas sobre las que la entidad ha generado dinámicas de co-construcción y transferencia de saberes hasta el momento. La construcción de conocimientos compartidos entre actores de distintas naturalezas y procedencias interesados por temáticas muy concretas es lo que caracteriza la manera de trabajar de la organización. La disparidad temática de los distintos grupos de trabajo que hasta el momento han generado interés dentro del sector ha promovido cierta voluntad de modelizar la metodología del TIESS. Los promotores de la iniciativa se encuentran con el impulso de definir en que se caracteriza la forma de funcionar de la entidad y como esta otorga un alineamiento coherente a los distintos grupos de trabajo. A nuestro modo de entender esta reflexión puede estructurarse en torno a dos líneas generales: 1) por un lado la reflexión micro sobre cómo afrontar las problemáticas que inciden en la construcción de vínculo y transferencia de saberes entre actores, y por otro lado 2) una reflexión más macro sobre los retos del fomento de la economía social y solidaria y su conexión con las dinámicas de desarrollo territorial. Consideramos al TIESS un caso interesante porque en su práctica otorga importancia a promover la rentabilidad social de la transferencia de saberes sobre innovación social en ambos sentidos.

\section{Problemáticas comunes en la "co-construcción" del vínculo y la transferencia de saberes sobre innovación social}

Siguiendo a Fontan (2010) y Fontan et al. (2013), hay ciertas condiciones que se tienen que dar para asegurar una co-construcción útil y efectiva en términos de producir un conocimiento socialmente relevante. Se trata de condiciones para garantizar el proceso de transferencia que Fontan refiere a la investigación-acción partenarial y que pueden extenderse a la co-construcción de vínculo y transferencia sobre innovación social. Como puede observarse en la Tabla 1, estos retos ya identificados como factores constitutivos de una investigación-acción socialmente relevante, han tenido una consideración atenta en el desarrollo del TIESS.

En primer lugar, es necesario un nivel suficiente de confianza entre los actores con una actitud flexible y abierta a la producción de un aprendizaje colectivo. Una confianza que permita una orientación hacia el trabajo en equipo por parte de todos los actores que intervienen en el proceso (Fontan et al., 2013: 308). En esto, Fontan destaca como clave la existencia de un claro liderazgo. El liderazgo implica asegurar que las reglas de juego sean respetadas y que la co-construcción sea posible sin decantarse hacia una objetividad concreta. Para una organización como el TIESS esto quiere decir crear un ambiente en que la crítica siempre sea posible manteniendo a la vez una dirección clara y bien definida por lo que respecta a los objetivos de los procesos de construcción de saberes. Los grupos de trabajo requieren de temas bien definidos para que sean operativos, y dicha selección de temas implica necesariamente la relegación de otros.

También es necesario otorgar espacio y tiempo para la reflexión participada por parte de todos los actores implicados en el funcionamiento de la organización. Dicho ambiente no se crea fácilmente por el condicionamiento de muchos factores organizativos. Por ejemplo, la distinta disponibilidad de los actores para acudir a las reuniones y encuentros de la organización. Si los actores académicos disponen de más facilidades y proximidad, no solo a nivel geográfico, para acudir a acciones y eventos de reflexión y debate, es necesario que las organizaciones que fomentan la co-construcción y la transferencia planteen acciones correctoras como pueden ser debates descentralizados o formas de asistencia no presencial. La cuestión del tiempo también es un punto sensible. Los vínculos de confianza requieren de tiempo para ser consolidados. El paradigma de la transferencia de saberes es heredado del modelo según el cuál las organizaciones de transferencia adaptan avances del conocimiento para su aplicación industrial o mercantil. Dicho modelo, del que organizaciones como el TIESS no se pueden declarar totalmente desvinculadas, plantea la necesidad de cumplir con calendarios y presupuestos, y ofrecer productos resultantes de dichos procesos en tanto que pruebas de la transferencia. En este aspecto concreto, el reto asumido por el TIESS mediante la acción de los técnicos de transferencia que trabajan para la entidad, es la búsqueda de un cierto equilibrio entre los ciclos de vida de los proyectos y el ritmo más pausado de la construcción de vínculo entre actores socialmente creativos.

Otra cuestión clave en la co-construcción es asegurar la traducción de los lenguajes mediante los cuáles se tejen vínculos de conocimiento. Fontan describe que es necesario asegurar la comprensión y la inteligibilidad de todas las aportaciones por parte de todos los actores que toman parte del proceso de construcción del conocimiento (Fontan, 2010). Desde la perspectiva de una iniciativa como el TIESS la importancia otorgada a los lenguajes a través de los cuáles se ejerce la transferencia de conocimientos es relevante. Más allá de las herramientas clásicas desarrolladas por el medio académico, el modo en que el TIESS está dando progresivamente importancia a la generación de cápsulas audiovisuales, por ejemplo, nos informa de dicha preocupación. A parte de la preocupación por la emisión de un discurso inteligible dicha cuestión implica 
también considerar los canales a través de los cuáles los actores y el público objetivo de la transferencia de conocimientos se informa y comunica. Sin duda en este sentido el reto reside en la superación de un proceso que no se puede terminar mediante la generación de informes de investigación que luego tienen muy poca repercusión.

Otra condición necesaria para la co-construcción es cierta capacidad para mediar en situaciones de conflicto o de frágiles equilibrios de comprensión entre actores. Por lo que se refiere a la mediación, Fontan (2010) señala que es necesaria para facilitar el desarrollo de un compromiso compartido alrededor del proceso de investigación e intervención. La co-construcción implica de manera continua y renovada el trabajo de negociación y reformulación de los marcos de referencia. En este sentido la identificación del cometido del TIESS es un buen ejemplo de un aspecto concreto que requiere constantemente de un esfuerzo de mediación por parte de los líderes de la iniciativa. El propio nombre de la organización "Territorios innovadores en economía social y solidaria" plantea una síntesis compleja entre mundos aparentemente opuestos como son el de la innovación económica y el desarrollo socioterritorial. Por otro lado la expresión "Territorios innovadores" exige cierta reflexión al respecto del porqué el objeto de la organización son los territorios y no los actores. Los promotores de la iniciativa se encuentran de manera frecuente con la necesidad de remarcar - también entre algunos de sus miembros- que se trata de una organización que promueve el vínculo, la comunicación y la transferencia (liaison et transfer) y no se trata específicamente de una organización que promueva la investigación. Subrayar una y otra vez que la iniciativa se preocupa por la utilidad social de la investigación, por su circulación, forma parte del trabajo de mediación que realiza la entidad entre los diferentes tipos de actores que se relacionan en su práctica cotidiana. La comprensión de su objetivo organizativo o de su función en el ecosistema de innovación social, desde la perspectiva de los promotores de la iniciativa, se consigue mediante el acompañamiento de los actores y agentes interesados y la transferencia de saberes implícitos que este acompañamiento conlleva. El siguiente fragmento de la entrevista realizada al director del TIESS ejemplifica dicha perspectiva.

(...) Tenemos un compañero al que acompañamos, y a menudo en la transferencia también aprendemos por este mecanismo, por acompañamiento, mediante conocimientos implícitos, de manera no explicitada, el rol de la investigación es mirar lo que estás haciendo, estas apunto de hacer eso, mira tu gobernanza, mira tus fuentes de financiación, como animas tus cosas, la importancia de la movilización, tu quieres repetir, pero es importante que te des cuenta de tu gobernanza, y como vienen las otras cosas, Sistematizamos el conocimiento para poder mejorar, para poder evitar de volver a hacer los mismos errores, y para poder ir más de prisa. Entonces trabajamos con la investigación, para la sistematización, para hacer el agrupamiento de conocimientos, pero no hacemos investigación como tal. Podemos contribuir a desarrollar la investigación, a construir un partenariado. Podemos acompañarla. (Entrevista Vincent van Schendel - Director del TIESS)

Fontan (2010) destaca también como una condición importante de la investigación-acción partenarial evitar la instrumentalización de los actores. En el mismo sentido, la co-construcción del conocimiento, o si se quiere los procesos de generación de conocimiento compartido, implica evitar utilizar a los participantes como coartadas para la realización de un proceso completamente controlado por una de las partes. En los procesos de investigación partenarial existe la posibilidad que una vez facilitada la operación de la captación de datos nos encontremos de nuevo con una situación clásica de investigación. Por otro lado, en el sentido contrario, los investigadores pueden ser utilizados por los actores como consultores, para realizar una investigación a demanda, y una vez los resultados son producidos dejar de lado otro tratamiento que el que responde estrictamente a sus intereses particulares. Entidades como el TIESS también tienen riesgos por lo que respecta a la instrumentalización de su actividad. El imperativo de la sostenibilidad organizativa y la rendición de cuentas con las instancias que financian su actividad -siempre con una orientación política determinada- requiere de una observación crítica a los indicadores de evaluación con los cuáles se mide su actividad. Esto implica la necesidad de observar críticamente los indicadores de evaluación oficiales. También la consideración de la rendición de cuentas con todos los actores involucrados en la coconstrucción, no sólo con las instancias financiadoras de los proyectos. En definitiva, más allá de la posibilidad de instrumentalización política, en entidades como el TIESS que exploran la generación de vínculos sociales y la transferencia de saberes, la democratización del proceso de producción de conocimientos científicos es una cuestión crucial que no está ni mucho menos garantizada. 
Tabla. 1. Factores relevantes en la co-construcción de saberes promovida por el TIESS.

\begin{tabular}{|c|c|}
\hline $\begin{array}{l}\text { Condiciones de una investigación- } \\
\text { acción participativa } \\
\text { relevante }\end{array}$ & s en la co- \\
\hline Confianza y trabajo en equipo & $\begin{array}{l}\text { Historia de interlocución entre los actores de la economía social y el medio } \\
\text { académico. } \\
\text { - Organización en grupos temáticos bien definidos y con una dirección clara. }\end{array}$ \\
\hline $\begin{array}{l}\text { Espacio y tiempo para la con } \\
\text { del vínculo entre actores }\end{array}$ & $\begin{array}{l}\text { Consideración del condicionamiento de los factores organizativos } \\
\text { (disponibilidad horaria de los actores, estímulos laborales...). } \\
\text {-Acciones correctoras (debates descentralizados, formas no presenciales de } \\
\text { participación...). } \\
\text {-Búsqueda de equilibrio entre el ciclo de vida de los proyectos y los ritmos de } \\
\text { la construcción de vínculo. }\end{array}$ \\
\hline \begin{tabular}{|llll}
$\begin{array}{l}\text { Facilitar la traducción y lenguajes } \\
\text { comunes }\end{array}$ & & \\
\end{tabular} & $\begin{array}{l}\text { portancia de los lenguajes / formatos audiovisuales alternativos a l } \\
\text { ulgación académica típica. } \\
\text { onsideración de una comunicación multicanal y de un público objetivo. }\end{array}$ \\
\hline $\begin{array}{l}\text { Acciones de mediación en situaciones } \\
\text { de conflicto y de frágiles equilibrios de } \\
\text { comprensión }\end{array}$ & $\begin{array}{l}\text { Identificación de la construcción del vínculo que posibilita la transferenci } \\
\text { como principal cometido de la entidad. } \\
\text {-Preocupación por la utilidad social de la investigación partenarial. } \\
\text { - Acompañamiento de actores desde una perspectiva integral. }\end{array}$ \\
\hline $\begin{array}{l}\text { Evitar la instru } \\
\text { cctores y el proce }\end{array}$ & $\begin{array}{l}\text { crítica de los indicadores de evaluación de la transferenci } \\
\text { cuentas de las actividades con todos los actores involucrados, nc } \\
\text { nstancias financiadoras. }\end{array}$ \\
\hline
\end{tabular}

Fuente: Elaboración propia.

\section{EI TIESS frente a los desafíos contemporáneos del desarrollo territorial}

Los retos con los que se encuentra el TIESS informan también de las contradicciones de un mundo que pese a las condiciones que favorecen la circulación de información se encuentra con dificultades para fomentar el vínculo social y la transferencia de conocimientos. Un contexto en el que la fragmentación y el aislamiento social (Mingione, 1991) afectan no solo las vidas personales, sino que también la capacidad de los territorios, sus actores y las organizaciones en que se articulan, para definir su propia trayectoria de desarrollo.

Como destaca la literatura sobre el fomento de la economía social y solidaria (Eizaguirre, 2017; Fraisse, 2013; Garcia Jané, 2014) la vigilia, la observación y el análisis orientado a la sistematización y divulgación de conocimientos es el primer escalón de la valorización de los saberes y las experiencias de la economía social y solidaria. En el caso del TIESS la tarea de observación se orienta a identificar experiencias portadoras de dinámicas de innovación social y se nutre del trabajo desarrollado por las antenas regionales y los grupos de trabajo que participan de la red. Uno de los retos para el desarrollo territorial que se pone de manifiesto en este sentido es que en el contexto de la sociedad del conocimiento, la dificultad no se encuentra en la producción de información y en la identificación de "casos de éxito", sino más bien en la posibilidad de los territorios de crear y difundir conocimiento significativo y encontrar recursos (sobretodo el tiempo necesario) que permitan apropiarse de él y adaptarlo a sus propias realidades territoriales. En los procesos de desarrollo local mucho del trabajo que identifican los actores como clave tiene que ver con transferencia de saberes alrededor de las posibilidades democratizadoras de una visión plural de la economía. Este aprendizaje se basa en un proceso lento de apropiación que requiere de una visión multidimensional del territorio y las comunidades que lo habitan en tanto que instancias educadoras con sus dinámicas e historias particulares. En este sentido se puede subrayar que se trata de un proceso en el que no es posible replicar innovaciones de un contexto a otro, sino que la transferencia y co-construcción de conocimientos al respecto de iniciativas concretas aspira a la promoción de dinámicas particulares en cada territorio.

En consonancia con la perspectiva del desarrollo territorial integrado (Klein et al., 2016, Moulaert \& Nussbaumer, 2008) el TIESS se ha puesto en funcionamiento gracias a una red de antenas regionales ${ }^{11}$. Estas antenas buscan garantizar una conexión estrecha entre los trabajos desarrollados por el TIESS y las regiones, realizando una lectura de los proyectos específicos de desarrollo con los que cuenta cada territorio y su vínculo particular con la economía social. Cada una de estas antenas agrupa al sector de la economía social y

11 En 2016 la red contaba con ocho antenas regionales: Bas-Saint-Laurent, Gaspésie-Îles de la Madeleine; Centre-du-Québec, Estrie, Lanaudière, Laurentides, Mauricie, et Outaouais. 
las instituciones de educación superior del territorio. Entre las temáticas abordadas por las antenas regionales se encuentran cuestiones como la movilidad y el transporte en el medio rural o los medios de comunicación comunitarios y la cobertura mediática a nivel regional. También aparecen como clave para estos actores temáticas como el papel de la mujer en el relevo generacional agrícola, la seguridad y la soberanía alimentaria, los servicios de proximidad, el desarrollo de actividades comerciales en el seno de las organizaciones no gubernamentales o la economía digital, entre otras preocupaciones. No obstante, a pesar de la importancia contrastada de dichas antenas para el funcionamiento del TIESS una de las dificultades que encuentra la organización se relaciona precisamente con la distancia física y la jerarquía socio-espacial entre territorios. La jerarquía entre los actores de la ciudad central, que en el caso del TIESS es Montreal, y las regiones, se pone de manifiesto en la existencia de dificultades para comprender la finalidad de la acción de la entidad por parte de algunos de los actores que se encuentran más físicamente alejados del centro. Estas dificultades ponen de relieve la cuestión de que en la sociedad red (Castells, 2010), no solo las desigualdades inter-espaciales no han desaparecido, como se pudo creer en un principio, sino que se han intensificado con la emergencia de la metropolización (Veltz, 2017). Existen jerarquías entre territorios y actores que participan dentro de una misma red, incluso cuando se trata de una perspectiva solidaria. Tener en cuenta dichas asimetrías es uno de los retos que deben abordar las redes que se plantean la organización del vínculo y la transferencia de saberes sobre innovación social.

Finalmente, otra de las dificultades que se pueden identificar en el funcionamiento del TIESS tiene que ver con la generación de un vínculo constructivo entre actores encuadrados en las rutinas académicas y las inercias profesionales del desarrollo territorial. De manera paralela a las antenas regionales, los denominados "comités de veille" reúnen tres veces al año a investigadores y profesionales, así como una buena parte del equipo de trabajo del TIESS para hacer un análisis en profundidad de alguna de las tendencias percibidas sobre el terreno y dentro el medio académico. Los resultados de estas discusiones alimentan los trabajos en curso y los espacios en los que se construirá la transferencia de saberes en un futuro. Los consejeros en transferencia que trabajan contratados en el TIESS preparan y supervisan la operacionalización de dichos encuentros de manera periódica. En la misma línea también existe un consejo científico que asegura un rol consejero a las otras instancias y estructuras de trabajo del TIESS. Los miembros de dichos comités y del consejo científico provienen de los diferentes entornos y redes asociados al TIESS, integrando tanto a investigadores, como a miembros del sector asociativo o establecimientos de enseñanza superior. Sin embargo, a pesar de la atención otorgada a la vigilia científica sobre las actividades de la entidad, uno de los puntos débiles de dicha relación es el relativo poco peso que aún tiene la transferencia de conocimientos en el ámbito de las ciencias sociales por lo que respecta a la articulación de la carrera académica. Son varias las voces que destacan que aún los jóvenes investigadores, o los científicos sociales en etapas incipientes de sus carreras, no encuentran suficientemente reconocidas ni estimuladas sus actividades de transferencia. Esto ocurre a pesar de que una universidad como la UQAM, mediante su Service aux Collectivités, puede considerarse un referente de fomento de la transferencia en el ámbito de las ciencias sociales.

\section{Conclusiones}

A lo largo de este artículo hemos observado como una nueva manera de funcionar ha ganado peso a lo largo de las últimas décadas en ambientes comprometidos con el desarrollo territorial, la economía social y la investigación partenarial en el Quebec. Dicho cambio de paradigma, especialmente novedoso en el ámbito de las ciencias sociales, ha coincidido con un momento de crisis institucional y ofensiva neoliberal en el que se ha actuado contra las estructuras de desarrollo territorial existentes. Las estructuras que tradicionalmente se han dedicado al acompañamiento y la intermediación entre actores sociales fueron claves en su momento en la identificación del valor de la co-construcción participada entre la ciudadanía y los actores institucionales sobre el terreno como un aspecto crucial de los procesos de desarrollo territorial. A lo largo de este artículo hemos sostenido que el TIESS representa un interés renovado por garantizar la rentabilidad social de la coconstrucción.

El TIESS emergió en un momento de desmantelamiento de las estructuras de intermediación y acompañamiento en el ámbito del desarrollo territorial. En ausencia de estas estructuras, nuevas organizaciones nacidas como alianzas interterritoriales en favor de la transferencia de conocimientos sobre innovación social y desarrollo han tomado protagonismo. También nos encontramos con un cambio de paradigma hacia la co-construcción y la transferencia de saberes bajo el ideal normativo de la sociedad del conocimiento. Evitar que la sociedad contemporánea siga evolucionando hacia un "capitalismo del conocimiento" extremadamente elitista en la que los saberes se conviertan en un bien privado central requiere también de una voluntad política comprometida con el ritmo y la rentabilidad social de una coconstrucción y transferencia de saberes democratizadora. Para su funcionamiento entidades como el TIESS se encuentran con la necesidad de romper con la producción mercantilizada de saberes que en última instancia sirve solo a los intereses de una elite concreta. A lo largo del artículo hemos podido observar 
algunos de los desafíos contemporáneos por lo que respecta a la gestión de las expectativas, al trabajo cooperativo de construcción del conocimiento y a sus condiciones necesarias para promover procesos democratizadores.

Una de las limitaciones claras con las que cuenta la iniciativa del TIESS es el hecho que como entidad de transferencia de conocimientos no procede propiamente a la transferencia de innovaciones sociales. Las innovaciones sociales son siempre muy dependientes del contexto institucional y organizacional en el que se desarrollan y están condicionadas por las capacidades colectivas de las comunidades en las que se imbrican. El TIESS como entidad que se dedica a la transferencia de conocimientos puede transferir saberes al respecto de la co-construcción de innovaciones sociales. Su puesta en funcionamiento se dedica a difundir la actividad de entidades u organizaciones que desarrollan respuestas a retos de dinamización socioeconómica, pero estos procesos siempre deben ser adaptados a nuevos contextos.

Finalmente, una de las cuestiones de fondo que aparecen en el análisis de casos como el presentado es el reto de hacer inteligible una cuestión abstracta. El vínculo y la transferencia son conceptos que generan dudas entre muchos actores. No es posible corroborar su materialización a corto plazo. Dicho problema, como ha señalado la literatura específica sobre economía social y solidaria, afecta también la medición y parametrización de la economía social y solidaria y sus efectos democratizadores que requiere de atención y complejidad explicativa. A fin de cuentas, lejos de serializar y paquetizar la transferencia de innovaciones sociales, lo que puede considerarse actualmente como un verdadero reto del desarrollo territorial ante una crisis multidimensional o civilizatoria como la contemporánea, es la cuestión de organizar el vínculo entre grupos de personas que se plantean de manera cotidiana no abandonar la construcción compartida de un proyecto en común. Se trata de salir de una visión estrecha del conocimiento, que solo valora lo que puede ser medido a partir de criterios coherentes con el sistema económico dominante, y construir conocimientos adecuados al desarrollo de capacidades colectivas que permitan romper los círculos viciosos de la desvitalización de territorios sometidos a procesos de periferización y de marginalización.

\section{Referencias bibliográficas}

Beaudin, É.; Laplante, R. \& Lizée, M. (2016) Canaliser les capitaux des caisses de retraite pour financer le développmnet territorioal et l'économie social. Analyse d'expériences nord-américaines. Montréal. Institut de recherche en économie contemporaine (IRÉC) et Territoires innovants en économie sociale et solidaire (TIESS).

Bouchard, M. (2013) Innovation and the Social Economy: The Québec Experience. Toronto, University of Toronto Press.

Bouchard, M. J.; Lévesque, B. \& St-pierre, J. (2005) Modèle québécois de développement et gouvernance: entre le partenariat et le néolibéralisme? Les Cahiers Du CRISES, ET0505.

Bouchard, M. J. \& Lévesque, B. (2010) Économie sociale et innovation. L'approche de la régulation, au cœur de la construction québécoise de l'économie sociale. Les Cahiers Du CRISES, ET1103.

Castells, M. (2010) Globalisation, Networking, Urbanisation: Reflections on the Spatial Dynamics of the Information Age. Urban Studies, 47(13), pp. 2737-2745. https://doi.org/10.1177/0042098010377365.

Chaves Ávila, R. \& Demoustier, D. (2013) The Emergence of Social Economy in Public Policy. An international perspective. (CIRIEC, Ed.). Peterlang, International Academic Publishers.

Chaves-Avila, R. \& Savall-Morera, T. (2019) The Social Economy in a Context of Austerity Policies: The Tension Between Political Discourse and Implemented Policies in Spain. Voluntas, 30 (3), pp. 487-498. https://doi.org/10.1007/s11266-018-00075-3.

Dagnino, R. (2012) Elementos para uma avaliação das incubadoras universitárias de cooperativas, Otra Economía, 6 (11), pp. 184-197.

Dubeux, A. (2013) Technological incubators of solidarity economy initiatives: a methodology for promoting social innovation, in Brazil. In Moulaert, F.; MacCallum, D.; Mehmood, A.; \& Hamdouch, A.; (Eds), The international Handbook of Social Innovation: Collective Action, Social Learning and Transdisciplinary Research. Cheltenham, UK: Edward Elgar, pp. 299-300.

Dumais, L. (2011) La recherche partenariale au Québec: tendances et tensions au sein de l'université, SociologieS [En ligne], Dossiers, Les partenariats de recherche, mis en ligne le 18 octobre 2011, consulté le 25 octobre 2013. URL: http://sociologies.revues.org/3747.

Eizaguirre Anglada, S. (2017) De la innovación social a la economía solidaria. Claves prácticas para el desarrollo de políticas públicas. CIRIEC-España, Revista de Economia Pública, Social y Cooperativa, (December 2016), pp. 201-230.

Eizaguirre, S.; Pradel, M.; Terrones, A.; Martinez-Celorrio, X. \& Garcia, M. (2012) Multilevel Governance and Social Cohesion: Bringing Back Conflict in Citizenship Practices. Urban Studies, 49 (9), pp. 1999-2016.

Fernandez, A.C.; Novy, A. \& Singer, P. (2013) The linkages between popular education and solidarity economy in Brasil: An historical perspective, in Moulaert, F.; MacCallum, D.; Mehmood, A.; \& Hamdouch, A.; (Eds),. The International Handbook of Social Innovation: Collective Action, Social Learning and Transdisciplinary Research, Cheltenham, UK: Edward Elgar, pp: 384-396. 
Fontan, J.-M. (2010) Recherche partenariale en économie sociale: Analyse d'une expérience novatrice de coproduction des connaissances. La Revue de l'innovation : La Revue de l'innovation Dans Le Secteur Public, 15 (3).

Fontan, J.-M.; Harrisson, D. \& Klein, J.-L. (2013) Partnership Based Research: Coproduction of Knowledge and Contribution to Social Innovation, in Moulaert, F.; MacCallum, D.; Mehmood, A.; \& Hamdouch, A. (Eds) The international handbook on Social Innovation. Collective action, Social Learning and Transdiciplinary Research. Cheltenham (UK) - Northampton, MA, USA: Edward Elgar. (pp. 308-319).

Fontan J.-M.; Klein, J.-L. \& Bussières, D. (2014) Le défi de l'innovation sociale partagée. Savoirs croisés, Québec, Presses de l'Université du Québec.

Fontan, J.-M.; Klein, J.-L.; Caillouette, J.; Doyon, M; Lévesque, B.; Tremblay, P.-A.; Tremblay, D.-G. \& Trudelle, C. (2017, daté de 2014) Vers de nouveaux modèles d'action en développement territorial : l'expérimentation à l'échelle locale de la transition vers le «buen vivir». Économie et solidarités. Vol. 44, No 1-2, pp. 84-102. DOI:10.7202/1041606ar

Fraisse, L. (2013) The social and solidarity-based economy as a new field of public action: a policy and method for promoting social innovation, in Moulaert, F.; MacCallum, D.; Mehmood, A.; \& Hamdouch, A. (Eds) The international handbook on Social Innovation. Collective action, Social Learning and Transdiciplinary Research. Cheltenham (UK) - Northampton, MA, USA: Edward Elgar.

Garcia Jané, J. (2014) La caixa d'eines de l'economia social i solidària: reparar i construir. In Guia d'economia social $i$ solidària per a l'administració local. Barcelona: Diputació de Barcelona (pp. 21-46). Gillet, A. \& D.-G. Tremblay (2017) (Eds) Les recherches partenariales et collaboratives. Rennes, Presses universitaires de Rennes

GUNi (2017) Higher Education in the World 6; Towards a Socially Responsible University: Balancing the Global with the Local. Global University Network for Innovation (GUNI) (Available at http://www.guninetwork.org/).

Heck, I. (2017) Du soutien à l'action à la recherche et development. Bilan des activités du volet de recherche interne à Parole d'ExcluEs (2013-2017). Montréal: Incubateur universitaire Parole d'ExcluEs.

Huot, G.; Gruet, É.; Dahmani, S.; Léonard, M. \& De, S. (2016) La franchise sociale comme stratégie de changement d'échelle, 55. Montréal: TIESS. Retrieved from http://www.tiess.ca/wp-content/uploads/2016/06/Franchisessociales TIESS Juin2016.pdf.

Klein, J.-L. (2017a) Social innovation, Universities and the Quest for Social Transformation. in GUNi, Higher Education in the World 6; Towards a Socially Responsible University: Balancing the Global with the Local. Global University Network for Innovation (GUNI) pp: 165-178 (Available at http://www.guninetwork.org/)

Klein, J.-L. (2017b) L'innovation Sociale Au Coeur de l'analyse de La Transformation Sociale. La Programmation Scientifique Du CRISES 2014-2020. Retrieved (https://crises.uqam.ca/upload/files/publications/etudestheoriques/CRISES_ET1703.pdf).

Klein, J.-L. \& J.M. Fontan (2014) Introduction. in LÉVESQUE, B., FONTAN, J.-M. \& J.-L. KLEIN (2014) Linnovation sociale: les marches d'une construction théorique et pratique. Québec, Qc, Presses de l'Université du Québec; pp. 110.

Klein, J.-L.; Fontan, J.-M. \& Harrisson, D. (2010) L’Innovation Sociale Dans Le Contexte Du «Modèle Québécois. The Philantropist, 23 (3), pp. 235-246.

Klein, J.-L.; Fontan, J.-M. \& Harrisson, D. (2009) L'innovation sociale au Québec : un système d'innovation fondé sur la concertation, Montréal, Cahiers du CRISES, $\mathrm{n}^{\circ}$ ET0907.

Klein, J.-L.; Fontan, J.-M.; Harrisson, D. \& Lévesque, B. (2013) The Quebec Model: a social innovation system founded on cooperation and consensus building, in Moulaert, F.; MacCallum, D.; Mehmood, A.; \& Hamdouch, A. (Eds) The international handbook on Social Innovation. Collective action, Social Learning and Transdiciplinary Research. Cheltenham (UK) - Northampton, MA, USA: Edward Elgar. (pp. 371-384).

Klein, J.-L.; Fontan, J.-M.; Harrisson D.; \& Lévesque, B. (2017) El efecto transformador de un ciclo de innovaciones sociales en Québec. Dans Gaiger, LI.; \& Mendonça dos Santos, A. (dirs) Solidariedade Popular e Emancipações. Sao Leopoldo RS, Editora Unisinos, pp :154-174.

Klein, J.-L.; Bussières, D.; Caillouette, J.; Doyon, M.; Fontan, J.-M.; Tremblay, D.-G.; Mai, C. (2015) Saint-Camille : Récit d'une expérience de co-construction de la connaissance. Montréal. Centre de recherche sur les innovations sociales, Cahiers Du CRISES, ET1505.

Klein, J.-L.; Fontan, J.-M. \& Tremblay, D.-G. (2003) Mundialización, acción colectiva e iniciativa local en la reconversión de Montreal. EURE (Santiago), 29 (86), 69-88. https://dx.doi.org/10.4067/S025071612003008600004.

Klein, J.-L; Laville, J.-L.; Moulaert, F. (2014) L'innovation sociale. Toulouse: ERES. Sociologie économique. 256 p.

Klein, J.-L.; Pecqueur, B.; Koop, K. \& Soussi, S. A. (2016) (Dirs.) L'innovation locale à l'épreuve du global. Un défi pour les acteurs. Québec, Qc, Presses de l'Université du Québec, 211 p.

Klein, N. (2016) "40 Years Ago, This Chilean Exile Warned Us About the Shock Doctrine. Then He Was Assassinated." The Nation, september 21. https://www.thenation.com/article/40-years-ago-this-chilean-exilewarned-us-about-the-shock-doctrine-then-he-was-assassinated/.

Le Bossé, Y.; Bourassa, B.; Chamberland, C. \& Vallerie, B. (2017) La contribution de l'initiative Parole d'Exclues au dévelopment du pouvoir d'agir individuel et collectif des citoyens du quartier Nord-Est de Montréal-Nord. Montréal. Incubateur universitaire Parole d'ExcluEs.

Lévesque, B. (2016) Économie Sociale et Solidaire et Entrepreneur Social: Vers Quels Nouveaux Écosystèmes?, Revue Interventions Économiques (En Ligne), 54, pp. 0-44. 
Lévesque, B.; Fontan, J.-M. \& Klein J.-L. (2014) L'innovation sociale: les marches d'une construction théorique et pratique. Québec, Qc, Presses de l'Université du Québec, 474 p.

Longtin, D. (2010) Revue de la littérature: la recherche-action participative, le croisement des savoirs et des pratiques et les incubateurs technologiques de coopératives populaires, Montréal, Centre de recherche sur les innovations sociales, Cahiers Du CRISES, ET1102.

Lussier, M. (2016) Pour des partenariats réussis en culture ! Stratégies de mobilisation des acteurs territoriaux. Montréal, Culture pour tous, Territoires innovants en économie sociale et solidaire (TIESS) \& Service aux collectivités UQAM, 24 p.

Mingione, E. (1991). Fragmented Societies. Oxford: Blackwell.

Moulaert, F. \& Nussbaumer, J. (2008) La logique sociale du développement territorial. Québec, Presses de 1'Université du Québec.

Novy, A.; Swiatek, D. C. \& Moulaert, F. (2012) Social Cohesion: A Conceptual and Political Elucidation.” Urban Studies, 49, pp. 1873-89.

Oosterlynck, S.; Kazepov, Y.; Novy, A.; Cools, P.; Barberis, E.; Wukovitsch, F.; Sarius, T. \& Leubolt , B. (2013) The butterfly and the elephant: local social innovation, the welfare state and new poverty dynamics. ImPRovE Discussion Paper No. 13/03. Antwerp: Herman Deleeck Centre for Social Policy - University of Antwerp.

Parés, M.; Ospina, S. \& Subirats, J. (2017) Social Innovation and Democratic Leadership. Communities and Social Change from Below. Cheltenham (UK) - Northampton, MA, USA: Edward Elgar Publishing.

Pecqueur, B. \& Itçaina, X. (2012) Economie sociale et solidaire et territoire : un couple allant de soi ?. RECMA, 325 (3), pp. 48-64. https://doi.org/10.7202/1017421ar.

Pozzebon, M. (2015) Tecnologia Social: A South American View of the Regulatory Relationship between Technology and Society. In De Vaujany, F.-X.; Mitev, N.; Lanzara, G. F.; Mukherjee, A. (Eds), Materiality, Rules and Regulation, Basingstoke: Palgrave Macmillan, pp. 33-51.

Roy, M. avec la collaboration de M.-J. Bouchard, É. Fortin, É. Gruet, G. Huot \& M. Vézina (2016) Synthèse de connaissances: L'entrepreneuriat social et l'enterprise sociale. Montréal. Territoires innovants en économie sociale et solidaire (TIESS).

Torre, A. (2015) Théorie du développement territorial, Géographie, économie, société, vol. 17, no 3, p. 273-288.

Vaillancourt, Y. (2019) De la co-construction des connaissances et des politiques publiques, SociologieS [En ligne], Dossiers, Savoirs savants, savoirs d'action et politiques publiques, mis en ligne le 23 mai 2019, consulté le 11 novembre 2019. URL : http://journals.openedition.org/sociologies/11589.

Veltz, P. (2017) La Société hyper-industrielle - Le nouveau capitalisme productif. Paris, Seuil. 